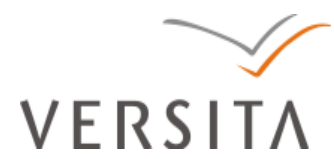

Folia Oeconomica Stetinensia

DOI: 10.2478/v10031-011-0038-6

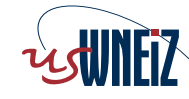

Wydzial Nauk Ekonomicznych i Zarządzania

Uniwersytetu Szczecińskiego

\title{
ON CERTAIN ANALOGIES BETWEEN THE LAWS OF QUANTUM MECHANICS AND RULES OF AN ENGLISH AUCTION
}

\author{
Ewa Drabik, Prof. \\ Faculty of Management \\ Warsaw University of Technology \\ Narbutta 85, 02-524 Warsaw \\ e-mail: ewa.drabik@poczta.fm
}

Received 27 September 2011, Accepted 29 December 2011

\begin{abstract}
It is a self-evident truth that nowadays a growing number of economic phenomena is described by means of physics methods. The most frequent theories derived from physics and applied to economy are: (1) the universal gravitation law and (2) the first as well as the second law of thermodynamics. The methods of static physics are applicable also to the theory of financial markets. In this case it is assumed that the financial market is composed of single participants interacting as a system of particles. Such approach is associated with a model of financial market otherwise known as a minority game. It is postulated that the process of securities and money allocation is performed on the basis of prices fluctuation, where - if a vast majority of investors tend to purchase goods or services - the sale constitutes a more profitable option, and vice versa. The players who end up on minority side win. At the end of the XX century the economy commenced to apply the laws of quantum mechanics. These laws proved to be useful, in particular when attempting to generalize game theory, which resulted in quantum games. The aim of the paper is to compare the rules and auction mechanisms with selected laws of quantum mechanics. This paper aims also to introduce the basic concepts of quantum mechanics to the process of economic phenomena modeling. Quantum mechanics is a theory describing a behaviour of microscopic objects and is grounded on the principle of wave-particle duality. It is assumed that quantum-scale objects at the same time exhibit both wave-like and particle-like properties. The key role in quantum mechanics is played by: (1) the Schrödinger equation describing the probability amplitude for the particle to be found at a given position and at a given time, as well as (2) the Heisenberg uncertainty principle stating that a certain pair of physical properties may not be simultaneously measured to arbitrarily high precision.
\end{abstract}

Keywords: quantum mechanics, quantum games, English auction.

JEL classification: C70, C72, D44, G14. 


\section{Introduction}

A significant number of economic models which were created within last years turn their attention to the laws of quantum physics. The list of the most common theories employed for economic purposes includes i.a.: the law of gravity, the principles of static physics, the first and second law of thermodynamics, as well as the laws of quantum mechanics which have achieved their greatest popularity within the last decade. Since 1960s the gravitational models have been applied in many branches of economic sciences, e.g. to describe relations between economic centers or characterize an international trade exchange. According to the rules of thermodynamics the increase in internal energy of a system is the energy that flows in a heat minus the energy that flows out as the work that the system performs on its environment, which fact is universally applied to the theory of economic equilibrium expressed in a variety of ways. The problem of equilibrium has been already addressed by a wide spectrum of prominent scientists, such as A. Smith, A. Cournot, W. Pareto, J. Tinbergen, P.A. Samuelson, J.R. Hicks, R. Selten, J.F. Nash, R. Lucas, etc.

The methods adopted by static physics, with a special focus placed on electromagnetic forces, are applicable to the theory of financial markets. In this case the financial market is defined as a system composed of several individual entities cooperating upon electromagnetic principles. This approach gives rise to a certain model of financial market, otherwise known as minority game. In the case of minority game the allocation of securities and funds is conditioned exclusively upon the fluctuation of prices, where the higher tendency to purchase goods and stocks results in the sale being more profitable, and vice versa. Thus, players from a minority group gain a prevailing position ${ }^{1}$.

It was only the end of the XX century when the laws of quantum mechanics commenced to be applied to quantum mechanics. The laws concerned proved extremely useful especially while attempting to generalize a classical game theory, which resulted in the creation of quantum games $^{2}$. Quantum mechanics discovered in 1926 is a theory describing the behaviour of microobjects. Its main postulate is a wave-particle duality. It is assumed that each microobject exhibits at the same time wave and particle properties. The key role in quantum mechanics is played by Schrödinger equation which allows to determine the probability range within which a microobject may be fund at a particular state as well as Heisenberg uncertainty principle stating a fundamental limit on the accuracy with which certain pairs of physical properties of a particle can be simultaneously determined. The aim of the work is to compare auction mechanisms with quantum mechanics. The auction theory is one of the most prolific theories 
within the field of economy, where the auction may be compared to the game. As a result, auctions are more and more frequently analyzed in the light of the game theory. The paper will present certain analogies between the mechanisms governing the auction, identified as a game, and the principles of quantum mechanics.

The work is organized in the following manner: the second part thereof introduces basic information on quantum mechanics, Schrödinger's cat thought experiment and Heisenberg uncertainty principle, as well as the suggestions concerning the applicability of the theoretical background provided. The third part supplies an example of a simple, two-person quantum game with a special emphasis placed on the description of auction functioning by means of quantum mechanics laws. A variety of analogies between the auction and quantum rules will be specified.

\section{Basic postulates on quantum mechanics}

\subsection{Elementary information}

Quantum mechanics is a theory desribing the behaviour of particles at an atomic level. The most noteworthy feature thereof is a wave-particle duality, according to which photons, electrons, light and matter exhibit both wave and particle properties. An object, e.g. an electron, may be treated as a particle due to its spatial qualities and, at the same time, as a wave due to its manner of changing location in the space. Within the framework of this article the particles will be referred to as microobjects.

Quantum mechanics states that all the natural phenomena may be described in approximation; each measured value is mistake-prone and falls within a specific range of probability. Uncertainty and inaccuracy do not result from the fact that measuring devices are imprecise, but rather from the very character of natural phenomena. It should be emphasized that quantum mechanics is subject to pure determinism, which refers, however, exclusively to the space of states. The phenomena within a physical space are not conditioned deterministically, where coordinates of microobjects may not be specified with arbitrarily high precision.

Quantum physics abolished a division into the observer and observable, which is considered as its main achievement. Whereas in classical physics an observer has no influence on the state of the measured object, quantum mechanics assumes that microobjects may be located at several places simultaneously until they are measured. Their connection may be so strong, which state is known as quantum entanglement, that - irrespective of the distance - they may behave as one entity. This property has its own "economic" analogy, since the branch offices of a significant 
corporation may be placed in separate corners all over the world. Despite the distance they constitute one "body".

The basis for quantum mechanics is constituted by the space of states of a given physical system, being a complex Hilbert space. The state of the system in quantum mechanics is represented by a vector space normalized to the unity. It is the so called pure state. On the other hand, scalar product is used for determination of probability of any physical value.

The main postulate of quantum physics, i.e. wave-particle duality, assumes that each microobject is at the same time characterized by a wave function as well as exhibits properties attributable to particles, i.e. has its own mass which may be localized at a particular state. Other parameters characterizing the state and features of microobjects are, except the location, quantum numbers. They describe energetic levels of a microobject. In quantum mechanics a key role is played by a Planck's constant. The energy of a given system may be changed in a linear manner, which means that the energy may take on randomly close values. In quantum mechanics the energy may take on exclusively discrete values equal to a total number of elementary portions of energy quanta $\varepsilon_{v 0}, \varepsilon_{v}=k \varepsilon_{v 0}, k=0,1, \ldots 3$

Theory of quanta is a generalization of classical physics. It is a broader and more comprehensive theory applicable both to atoms and planets. Whenever the mass of objects is significant and the wavelengths - in comparison to the distances under study - are short, all the phenomena referring to the evolution of those objects resulting from the theory of quanta are based on Newtonian mechanics or classical electrodynamics. The term "mass" may refer to countries, economic regions, provinces, cities, etc. The mass measure, depending on the problem analyzed, may be: a number of households, population of a given country, number of warehouses or shops, etc.

\subsection{Schrödinger equation and its applicability to economy}

The key issue in quantum mechanics is the system evolution which may take two forms. The first one is deterministic evolution described by a unitary operator. In this case the state of the system changes in a predictable way. The second way of evolution is of random nature and may be observed during the measurement. One possible prediction is the determination of probability with which a system may be found in a given state. In quantum mechanics a key role is played by a wave function which constitutes a basic characteristic of quantum objects. The function concerned defines the wave amplitude. Let $\Psi(x, y, z, t)$ denote a wave function, where $x, y, z$ are coordinates of an object located within the space $\mathfrak{R}^{3}, t$ is time. The square of 
function $\Psi$ denotes the probability density of finding a microobject at the moment $t$ in a given point described by coordinates $(x, y, z)$. From a formal point of view $|\Psi|^{2}$ satisfies the following condition:

$$
\Psi^{*} \Psi=|\Psi|^{2}=p
$$

where

$$
\begin{aligned}
& \Psi^{*} \text { - a wave function coupled to } \Psi, \\
& p \text {-denotes probability. }
\end{aligned}
$$

As a result, one may assume that the probability density of a microobject which is to be found within a given space, whose volume amounts to $\Delta V=\Delta x \Delta y \Delta z$, is equal to:

$$
P=p \cdot \Delta V=p \cdot \Delta x \Delta y \Delta z
$$

Thus, a wave function corresponds to the probability density of finding a particle in a given place at a given time. It is not worth searching the object at places, where the square of the absolute value of the probability density equals $0^{4}$. The wave function is a solution to a differential equation, otherwise known as Schrödinger equation, which takes the form:

$$
i \hat{h} \frac{\partial \Psi}{\partial t}=-\frac{\hat{h}}{2 m} \bar{\Delta} \Psi+U(x, y, z, t) \Psi,
$$

where

$\Delta$ - Laplace operator, i.e. $\bar{\Delta} \Psi=\frac{\partial^{2} \Psi}{\partial x^{2}}+\frac{\partial^{2} \Psi}{\partial y^{2}}+\frac{\partial^{2} \Psi}{\partial z^{2}}$,

$m$ - a mass of the microobject, $U(x, y, z, t)$ is potential energy of a microobject depending on its localization,

$\hat{h}=\frac{h}{2 \pi}, h-$ the Planck's constant $\left(h=6,626 \cdot 10^{-34} J \cdot s\right), i=\sqrt{-1}$ is the imaginary unit.

It is additionally assumed that the function $\Psi$ is finite and continuous. It is noteworthy that economic systems are subject to business trades. Therefore, one may ascribe to them wave-like properties. They have a certain mass. In general, the wavelength associated with trade cycles is comparable to "units" defining the mass of an economic system. Thus, the application of quantum mechanics to economy seems logical and plausible. It is noteworthy that E. Schrödinger constructed its equation pertaining to the microworld on the basis of the laws applicable to the macroworld, especially principles of thermal waves propagation. Quantum effects become more and more significant whenever the wavelength associated with the object is comparable with the distance, which is quite typical in economic systems. The wave function may seem useful 
for the purpose of forecasts and analyses, since it allows for the determination of probability density of finding a given object within a space $(x+\Delta x, y+\Delta y, z+\Delta z)$. Schrödinger equation may be applied to the forecasts of economic phenomena, whose evolution proceeds in a chaotic manner, is not illustrated by a unified curve, i.e. its trajectory reflecting the system evolution is an attractor, also known as a strange attractor ${ }^{5}$. Forecasting future values in the event of such phenomena is rather difficult. In the case of strange attractors only short-term forecasts are possible. Therefore, instead of short-term forecasts, it is recommended to calculate the probability density with which a given system will be found within a specific space $(x+\Delta x$, $y+\Delta y, z+\Delta z$ ), which is possible by way of the wave function. This method may be treated as an alternative to the current forecasting procedures. Whereas traditional forecasting procedures often prove to be inappropriate (since they generate significant mistakes), Schrödinger equation allows for the identification of a "space" within which an object may be detected in the future with the highest probability. The economic version of the equation concerned is not easy to be attained, nevertheless feasible. Firstly, an economic equivalent of the Plack's constant should be found. In selected cases one may employ the Planck's constant. If, however, the phenomenon under study refers to prices or money, the Planck's constant may be replaced by one grosz, one cent, etc. Depending upon the problem concerned, it may take the form of one gram, one item, etc. Secondly, if the trajectory illustrating the evolution of an economic system is structured chaotically, it should be specified where (i) the attractor corresponding to the trajectory in question as well as (ii) the current coordinates determining the object's position are located. An attempt to find future values of the analyzed phenomenon located beyond the attractor's region may prove purposeless. In order to prepare the prognosis a method of "the closest neighbours" should be applied. The complexity of economic phenomena makes the presentation of the wave function $\Psi$ most difficult. In order to create this function one may apply the physical methods ${ }^{6}$. Having overcome all the difficulties associated with the specification of all variables constituting equation (3), one may attempt to arrive at an expected solution, i.e. find the location of the space $(x+\Delta x, y+\Delta y, z+\Delta z)(\Delta x, \Delta y, \Delta z \rightarrow 0)$, for which the square of an absolute value of function $\Psi$ will be the biggest. The location of this space will illustrate the best prognosis of the examined economic phenomenon.

\subsection{Cat Schrödinger's thought experiment}

A failure to predict events during the transition from state to another was expressed by E. Schrödinger in 1935. He gave an example of a cat imprisoned in a sealed box which after 
being exposed to radioactive substance may either die or remain alive. The chance that it will survive amounts to 50 per cent. According to Schrödinger, quantum mechanics implies that the cat remains both alive and dead (to the universe outside the box) until the box is opened, which may be described as follows:

$$
\left.\left.\left|\Psi_{\text {cat's }}\right\rangle=\alpha \mid \text { alive }\right\rangle+\beta \mid \text { dead }\right\rangle
$$

where $|\alpha|^{2}+|\beta|^{2}=1$.

Having opened the box, i.e. having measured the system, an external observer will find the cat either alive or dead. At that moment the "cat state" is reduced to one of the possibilities: $\mid$ alive $\rangle$ or $\mid$ dead $\rangle$. The experiment may be made even more complex by placing an internal observer in one of the box corners who will watch the situation from the inside. The "cat state" $\left|\Psi_{\text {cat's }}\right\rangle$ is observed and then reduced to one of its components: $\mid$ alive $\rangle$ or $\mid$ dead $\rangle$, which may be visualized as the state of: | happiness $\rangle$ or $\mid$ sadness $\rangle$, respectively. On the other hand, an external observer describes the entire system containing an internal observer in the form of a linear superposition:

$$
\left.\left.\left.\left.\left|\Psi_{\text {cat's }}\right\rangle=\alpha_{1} \mid \text { alive }\right\rangle \otimes \mid \text { happiness }\right\rangle+\beta_{1} \mid \text { dead }\right\rangle \otimes \mid \text { sadness }\right\rangle
$$

where $\left|\alpha_{1}\right|^{2}+\left|\beta_{1}\right|^{2}=1$.

The external observed will reduce the superposition concerned only after opening the sealed box. The idea is that whereas an external observer perceives the "cat state" as a superposition of two states (dead-alive), the internal observer may experience either happiness due to the cat's survival or sadness due to its death.

\subsection{Heisenberg uncertainty principle and summary of basic postulates of quantum mechanics}

A next important rule of quantum mechanics is the Heisenberg uncertainty principle which states a fundamental limit on the accuracy with which certain pairs of physical properties of an object can be simultaneously known. It is applied both to classical and quantum physics and associated with the accuracy of measurement. The measurement in physics is defined as the process of interaction between the gauging device and the object examined, as a result of which certain information as to the object's features is obtained. In many circumstances the 
measurement has a significant impact on the course of the analyzed process and serves for evaluation of a variety of phenomena.

Heisenberg uncertainty principle states that the product of the uncertainties in position and momentum is always equal to or greater than one half of the reduced Planck's constant (or its multiple value). The above rule is described by the following inequalities:

$$
\Delta x \Delta p_{x} \geq \hat{h} / 2, \quad \Delta y \Delta p_{y} \geq \hat{h} / 2, \quad \Delta z \Delta p_{z} \geq \hat{h} / 2
$$

where

$\Delta x, \Delta y, \Delta z$ denote intervals where the object may be localized,

$\Delta p_{x}, \Delta p_{y}, \Delta p_{z}$ denote projections of the momentum vectors on $x, y, z$ plane.

Symbols $\Delta x, \Delta y, \Delta z, \Delta p_{x}, \Delta p_{y}, \Delta p_{z}$ refer to the root mean square deviation of the position from its mean and the root mean square deviation of the momentum from its mean, i.e. $\Delta x=\sqrt{\left\langle\Delta x^{2}\right\rangle}=\sqrt{\left\langle x^{2}\right\rangle-\langle x\rangle^{2}}$, where $\langle x\rangle$ denotes a mean value universally applied in physics. The inequalities indicated in point (6) imply that position-momentum uncertainty product is always smaller than the Planck's constant.

Similar relations are to be found in classical physics while describing a wave-like movement in reference to macroobjects. In general, one may say that the product of canonically coupled uncertainties (e.g. a pair $\left[p_{x}, x\right]$ is canonically coupled) is not smaller than the Planck's constant. Uncertainty relations for the position and the range in momentum entail that it is impossible to determine accurately both the position and the direction and speed of an object at the same moment. Due to this property the notion of a phase line (trajectory) in the phase space may not be precisely defined, since it fails to be described by any "specifically determined line" evolving over time, but rather is characterized by a specific "blurred" region. Analogical situation may be applied to a wide spectrum of economic phenomena whose trajectory evolves in a chaotic manner. On the same token, all prognostic procedures and statistical methods used in economy may be compared to physical gauging devices in physics.

To recapitulate, all the basic postulates of quantum mechanics in physics may be captured as follows:

1) A system is completely described by a state function.

2) A physical system is to be found in a specific state with certain probability.

3) A linear operator is introduced for each dynamic value.

4) The function of state satisfies Schrödinger equation. 
5) Each physical object is characterized by a wave function whose square denotes the probability density of finding an object concerned in a given space.

6) A quantum object exhibits all particle-like properties, i.e. has its own mass, wave of a certain frequency, is represented by the states which may be defined by means of parameters known as quantum numbers.

7) Properties characterizing a quantum object, e.g. energy, at a given moment do not have one specific property, but are defined by a set of possible eigenvalues which may be calculated from Schrödinger equation.

8) Heisenberg uncertainty principle results from the lack of computation of canonically coupled operators, e.g. position and momentum.

It is noteworthy that differences between classical and quantum physics are significant. The classical theory precisely determines the position of an object at a specific moment, i.e. defines its trajectory. However, so far the accuracy of such calculations has been neither experimentally confirmed nor denied. The quantum theory fails to mention anything about a specific position of a microobject in the space. Instead, it envisages the probability density with which a microobject may be found in the given space. The supremacy of quantum physics over classical theory resides in its experiential confirmability. According to classical physics all physical values may be measured with arbitrarily high precision. Uncertainty principle may be understood as a method establishing the measurement precision. The methods of classical physics may not be used for the creation of reality which goes far beyond the set of the described elements. The next section presents a sample quantum game as well as an attempt of finding certain analogies between the classical auction and rules of quantum mechanics.

\section{Analogies between an English auction and quantum mechanics}

\subsection{Two-players quantum games}

The theory of quantum games is an extension of a classical theory game. It has been developing since the beginning of XXI century. Quantum games differ from "classical" games in that in the latter case the basic unit of information is called a bit, whereas the former are based on qubits, where there is one additional dimension associated to the quantum properties. In quantum games the strategies may be entangled and the payout may be illustrated by complex numbers. On the contrary, classical games require that the payouts take the form of integer numbers. A sample quantum game is presented below. 
Example. Two players take part in a multi-stage game (composed of several stages). Prior to each stage they may, however do not have to, toss a coin in order to determine a respective payout matrix. Player 1 may use one of the four strategies: “T, T", "N, T", “T, N", "N, N", where „T" refers to the toss of a coin and " $N$ " means that the coin has not been tossed. Player 2, on the other hand, may use only two strategies " $\mathrm{T}$ " and "N". The coin may be treated as a quantum object. The coin flipping may be deemed as a physical process corresponding to a certain unitary operation in the space of states, since the transfer of information can be perceived as a physical state. A deterministic choice (a given player does not throw a coin, but continues the game from the previous stage) corresponds to the identity transformation. The toss of a coin may be illustrated by the following game matrix:

where $i=\sqrt{-1}$.

$$
F=\left[\begin{array}{cc}
0 & 1 \\
-i & 0
\end{array}\right],
$$

Player 1 has the access to the complete table of quantum movements demonstrated in the matrix:

$$
U=\left[\begin{array}{cc}
\frac{-1-i}{2} & \frac{-\sqrt{2}}{2} \\
\frac{\sqrt{2}}{2} & \frac{-1+i}{2}
\end{array}\right]
$$

Tails means that player 1 plays $U$, heads corresponds to $U^{-1}$. If, however, the player fails to toss a coin, then:

$$
U^{-1} \circ I \circ U=I
$$

If player 2 tosses a coin and obtains a tails, the matrix takes the following form:

$$
U^{-1} \circ F \circ U=\left[\begin{array}{cc}
\frac{-\sqrt{2}+i \sqrt{2}}{2} & 0 \\
0 & \frac{\sqrt{2}-i \sqrt{2}}{2}
\end{array}\right] .
$$

The event of obtaining a heads results in the identity matrix.

The example shows that the quantum game strategies are more sophisticated and provide more opportunities than traditional mixed strategies in classical games ${ }^{7}$. The strategies adopted by player 1 are entangled, which means that respective quantum states attached to those strategies are to a certain extent related to each other. There are quantum games in which players 
are allowed to use two coins as well as games involving a higher number of participants ( $m>$ 2), among whom each, while selecting particular strategies, may toss a coin. The vast majority of classical games may be transformed to quantum games. The non-exhaustible list includes the Prisoner's Dilemma and the game called rock-paper-scissors. Generally speaking, assuming that two players may employ two strategies: $\sigma, \sigma^{\prime}$, their reasonable game tactics will lead to the state $\left|\Psi_{\text {out }}\right\rangle$. Following the theory of quanta one may calculate the probability $P_{\sigma \sigma^{\prime}}=\left|\left\langle\sigma \sigma^{\prime} \mid \Psi_{\text {out }}\right\rangle\right|^{2}$ with which the application of strategies $\sigma, \sigma^{\prime}$ will bring them to the state $\left|\Psi_{\text {out }}\right\rangle$.

Quantum games currently enjoy their increasing popularity. They are applied to, i.a. the theory of financial markets, the construction of quantum computers, quantum algorithms, cryptography, data transfer, or examination of decoherence. In the subsequent part the author attempts to present the auction as a quantum game.

\subsection{Auctions as quantum games}

Auctions are one of the oldest forms of trade and currency exchange. They are economic mechanisms aiming to effectively allocate goods and money within the "request for bids". The auction rules are arbitrarily established, therefore they may be treated as the game rules and the auction takes the form of a game. The auction is usually won by a purchaser, i.e. the bidder offering the highest price for the sold item. Auction trade revolves around versatile and nonstandard products, services and other goods. Auctions may be captured within several categories, however, one of the main classifications includes oral and written auctions. The group of oral auctions encompasses i.a. an English auction, where participants bid openly against one another, with each subsequent bid higher than the previous bid. An auctioneer may announce prices, bidders may call out their bids themselves, or bids may be submitted electronically with the highest current bid publicly displayed. If no competing bidder challenges the standing bid within a given time frame, the standing bid becomes the winner, and the item is sold to the highest bidder at a price equal to his or her bid. The other variation on oral auction system is a Dutch auction, also known as an open descending price auction. The auctioneer begins with a high asking price which is lowered until some participant is willing to accept the auctioneer's price. The winning participant pays the last announced price. This type of auction is in use by resale shops or in the case of perishable goods. Written auctions includes first-price sealed-bid auction and second-price sealed-bid auction. In the first-price sealed-bid auction, commonly used in tendering, all participants simultaneously submit sealed bids which are not known to other bidders. The highest bidder pays the price they submitted. In a Vickrey ${ }^{8}$ auction, also 
known as sealed-bid second price auction, bidders submit written bids without knowing the bid of the other people in the auction, and in which the highest bidder wins, but the price paid is the second-highest bid.

The remaining types of auction are still under development, and the auction theory is one of the most prolific fields interpenetrating the economic science and the game theory. The mathematical models of auctions are basically constructed on the grounds of the game theory ${ }^{9}$. Those models are mainly based on the classical probability theory which usually proves insufficient for determining certain subtleties associated with the functioning of auctions. Therefore, in the following part the author attempts to describe the notion of auction in the light of quantum mechanics. Certain analogies between auction rules and laws of quantum mechanics will be listed.

By and large, the purchaser is assumed to act rationally, which means that he or she will not buy any product whose price exceeds their reserve price (i.e. its evaluation price). Furthermore, one may assume that the terms "purchase" and "sale" are equivalent, since on the one hand, the sale implies the acquisition of money, and, on the other, in exchange for money one acquires goods.

Let us analyze the English auction involving $N$ purchasers (players 1, .., N) and one seller (player 0). Let $|\Psi\rangle_{k}{ }^{10}$ denote a Hilbert space vector defining the strategy or the state of the purchaser $k$. Function $\Psi(x)=\langle x \mid \Psi\rangle$ is the probability amplitude of the wave function $\Psi$. According to the quantum theory, whose main ideas were briefly presented in the third part, it is assumed that the square of the absolute value of the probability amplitude $|\langle x \mid \Psi\rangle|^{2}$ illustrates the probability density attributable to the measured property $x$ (e.g. price) of variable $X$, reflecting the state of the system. Due to the fact that the quantum theory is based on Hilbert space, the probability amplitude should be normalized to the unit in accordance with the following formula ${ }^{11}$ :

$$
\langle q \mid \Phi\rangle_{k}=\frac{\langle q \mid \Psi\rangle_{k}}{\langle\Psi \mid \Psi\rangle_{k}}, \quad(k=1, \ldots, N)
$$

where $\langle\Psi \mid \Psi\rangle=\int_{-\infty}^{+\infty} \Psi(x) \overline{\Psi(x)} d x$

Equation (7) denotes a normalized amplitude for the strategy applicable to purchaser $k$ bying the respective goods at price $q$. Expression $\left\langle p_{\text {in }} \mid \Phi\right\rangle_{0}=\frac{\left\langle p_{\text {in }} \mid \Psi\right\rangle_{0}}{\langle\Psi \mid \Psi\rangle_{0}}$ is a declaration of the 
seller (player 0) consisting in determining the reserve price (starting price) $p_{i n}$, i.e. the minimum price at which the good may be sold. The reserve price $p_{i n}$ is measurable.

According to quantum mechanics the probability density for a particular value of variable denoting the price of participant $k$ (player $k$ ) is equal to the square of the absolute value of the strategy normalized to the unit:

$$
P_{k}=\left|\Phi_{k}\right|^{2}=\frac{\left|\langle q \mid \Psi\rangle_{k}\right|^{2}}{\langle\Psi \mid \Psi\rangle_{k}}
$$

The auction is won by purchaser $k$ if his price is the highest, thus the probability of the state $\left|\Psi_{\text {out }}\right\rangle$ achieves its maximum value, i.e.:

$$
\frac{\left|\left\langle q_{1} \mid \Psi\right\rangle_{\text {out }}\right|^{2}}{\langle\Psi \mid \Psi\rangle_{\text {out }}} \leq \ldots \leq \frac{\left|\left\langle q_{k} \mid \Psi\right\rangle_{\text {out }}\right|^{2}}{\langle\Psi \mid \Psi\rangle_{\text {out }}}
$$

The equation mark suggests that the strategies of individual players may be entangled. In the theory of quanta the entanglement is defined as the correlated quantum state of two or more particles, or other quantum systems. In other words, the entanglement means that the quantum systems are interrelated even when kept at distance. In the case of auctions the assumption on the unified sale and purchase prices corresponds to quantum entanglement.

The auction consists in a dynamic transition from one state to another, i.e. in bidding higher prices:

$$
\left\langle\Phi_{\dot{\text { in }}} \mid \Phi_{\text {out }}\right\rangle=\sum_{i}\left\langle\Phi_{\dot{i}} \mid q_{i}\right\rangle\left\langle q_{i} \mid \Phi_{\text {out }}\right\rangle, \quad\left(q_{1} \leq q_{2} \leq \ldots \leq q_{k}\right)
$$

where $q_{i}$ denotes the price offered by purchaser $i$. They may be assumed to correspond to the states $e_{i}, e_{i} e_{j}=\delta_{i j}$, which can be presented in the simplified form as $\left|\Phi_{\text {out }}\right\rangle\langle i \mid j\rangle=\delta_{i j}$.

Comment. Quantum mechanics indicates that the transition from state $\mathfrak{N}$ to state $\mathfrak{I}$ may be described as the sum of products of amplitudes denoting the transition from state $\aleph$ to the subsequent states until the final state $\mathfrak{I}$ is reached:

$$
\langle\aleph \mid \mathfrak{I}\rangle=\sum_{i}\langle\aleph \mid i\rangle\langle i \mid \mathfrak{I}\rangle
$$

The transaction is completed when one of the purchasers (purchaser $k$ ) bids the highest price which will not be outbid by other participants. In this way he wins the auction. Purchasers 
$1, \ldots, k-1(k \leq N)$ are excluded from a further game, since due to their low bids they may not reach the final stage $\left|\Phi_{\text {out }}\right\rangle$-according to the Pauli exclusion principle they should be removed. The above principle states that two microobjects may not occupy the same state simultaneously, which rule defines the stability of the matter, and in the case of auctions, determines the harmonious interaction.

Thus, the transaction concerned consists in the transition from initial strategies $|\Phi\rangle_{\text {in }}$ adopted by auction participants to the final stage $|\Phi\rangle_{\text {out }}$ :

$$
|\Phi\rangle_{\text {in }}=T|\Phi\rangle_{\text {out }}
$$

where:

$T=|p\rangle_{i n}+\sum_{i j}\left\langle q_{i} \mid i\right\rangle\left\langle i\left|T_{A}\right| j\right\rangle\left\langle j \mid q_{j}\right\rangle$ is the transaction operator,

$|p\rangle_{\text {in }}$ is the starting price or reserve price,

$T_{A}$ denotes the operator of transition between the states,

$q_{i}$ is the price bid in the auction.

The purchaser is very often unable to evaluate the good, i.e. specify the maximum price which should not be exceeded. This price may violate as a result of the obtained information. The situation concerned corresponds to the Heisenberg principle. In the event of the English auction involving many purchasers the uncertainty principle may be formulated as follows: the product of two dispersion values, i.e. purchase and sale, may not be lower than the Planck's constant. The Planck's constant $h$ corresponds to the player's minimum risk inclination. This principle is applicable to each pair of players $(i, j)$, i.e.:

$$
\Delta q_{i} \Delta q_{j} \geq c \cdot h \quad(i \neq j, i, j=0,1, \ldots, k),
$$

where $c$ is a certain constant, $c \geq 0$.

Furthermore, the rational strategy, known from the auction theory, consisting in a failure to bid the price above one's own valuation price $\langle q \mid \Phi\rangle_{i} \leq\left\langle q \mid \mathrm{v}_{i}\right\rangle$, where $\mathrm{v}_{i}$ is valuation of product $i$ is also consistent with the uncertainty principle. Similar analogies may be proliferated, however, at least one more deserves special attention, i.e. Schrödinger's cat thought experiment described in point 2.3. The state of the seller $(k=0)$ before the transaction completion (i.e. the measurement) may be described as follows:

$$
\left.\left.\left|\Psi_{0}\right\rangle=\alpha \mid \text { transaction completion }\right\rangle+\beta \mid \text { failure to complete the transaction }\right\rangle
$$

where $\left|\alpha_{1}\right|^{2}+\left|\beta_{1}\right|^{2}=1$. 
The state of purchaser $k(k=1,2, \ldots, N)$ may be presented as follows:

$\left|\Psi_{k}\right\rangle=\alpha_{1} \mid$ satisfaction from the transaction completion $\rangle \otimes \mid$ transaction completion $\rangle+$

$+\beta_{1} \mid$ dissatisfaction from a failure to complete the transaction $\rangle \otimes \mid$ failure to complete the transaction $>$

where $\left|\alpha_{1}\right|^{2}+\left|\beta_{1}\right|^{2}=1$.

The states specified in formulas (11) and (12) correspond to "diagnoses" of the external and internal observers in Schrödinger's cat thought experiment. When the auction is completed, the initial state $\left|\Psi_{k}\right\rangle_{\text {out }}$ is reduced to the state: $\mid$ transaction completion $\rangle$ or $\mid$ failure to complete the transaction $\rangle$, where purchaser $k(k=1, \ldots, N)$ experiences $\mid$ satisfaction $\rangle$ whenever the product is purchased on profitable conditions, or $\mid$ dissatisfaction $\rangle$ in the opposite case.

\section{Conclusions}

Unlike the classical probability theory, the theory of quanta makes the modeling of economic phenomena more precise and accurate. Furthermore, there is a wide range of analogies between quantum mechanics and social sciences. In both cases the detailed identification of an object requires a higher number of measurements (i.e. determination of waves of versatile frequencies). Only large uncertainties allow for the specific identification of an object. Non-null correlation of variables pertaining to specific economic phenomena proves the dependency which may be compared to the entanglement of systems in the theory of quanta. It is commonly known that the evolution of a quantum system is determined by a unitary operator affecting the state at an initial moment. In the event of the auction a similar role is played by the transaction operator expressed by formula (10). Uncertainty and inaccuracy associated with the description of a variety of economic phenomena do not result from the fact that statistical and prognostic methods are ineffective. Analogically, a complicated nature of quantum phenomena is more relevant than the accuracy of gauging devices. As a result, both economic and physical phenomena may be described (determined) in approximation, according to the uncertainty principle.

To conclude, it is worth quoting the words of Antoni Smoluk who, following the thoughts of J. Hozer, claims that physics is "a universal domain and the laws formulated within one branch of science may be successfully transferred to another field of knowledge"12. 


\section{Notes}

1 Challet et al. 2000.

2 Landsburg 2004.

3 Feynman et al. 1972.

4 Ibidem.

5 Compare: Schuster 1995.

6 See Feynman et al. 1972, pp. 288-290 and 299-301.

7 Landsburg 2004.

8 William Vickey in 1994 was awarded the Nobel Memorial Prize in Economics, among others for the work on the auction theory.

9 See Drabik 2007.

${ }^{10}$ Strategies $|\Psi\rangle_{k}(k=1, \ldots, N)$ are components of Hilbert space $\mathrm{H}$.

11 Piotrowski 2001.

${ }^{12}$ Miscellanea mikroekonometrii..., 2010, p 101.

\section{References}

Challet, D., Marsli, M., Zhang, Y.-C. (2000). Modeling Market Mechanism with Minority Game. Physica A - 276, 284-315.

Drabik, E. (2007). Aukcje w teorii i praktyce. Warszawa: Wydawnictwo SGGW.

Feynman, R.P., Leigton, R.B., Sands, M. (1972). Feynmana wyktady z fizyki. T. III. Mechanika kwantowa. Warszawa: Państwowe Wydawnictwo Naukowe.

Landsburg, S.E. (2004). Quantum game theory. Notices of the AMS, 51(4), 394-399.

Piotrowski, E.W. (2001). Dwoistość wartości kapitalu. Białystok: Preprint rePEc:sla: aekjkl:69PLv3.

Schuster, H.G. (1995). Chaos deterministyczny. Wprowadzenie, Warszawa: Wydawnictwo Naukowe PWN.

Smoluk, A. (2010). XL lat działalności profesora Józefa Hozera czyli o metodologii ekonomicznej. In: Miscellanea mikroekonometrii. Ed. J. Hozer, Szczecin: Wydawnictwo Uniwersytetu Szczecińskiego. 